\title{
Rapid selective detection of ascorbic acid using graphene-based microfluidic platform
}

\author{
Goran M. Stojanović, Tijana Kojić, Mitar Simić, Aleksandra Jovanović-Galović, Bojan \\ Pavlović, Amaia Zurutuza, Luca Anzi, Roman Sordan
}

\begin{abstract}
In this paper we present a compact microfluidic platform for selective detection of ascorbic acid. The microfluidic chip was fabricated by xurography technique with microfluidic channel placed between the silver electrodes. To increase the conductivity of the platform and enhance electron transfer process, a graphene sheet was deposited in the gap between the electrodes. The suspension of tablets with ascorbic acid and a mixture of ascorbic acid and isomalt, a sugar substitute, were injected into the microfluidic channel. Measuring electrical parameters at the silver contacts, it was possible to successfully differentiate ascorbic acid from isomalt. The sensing mechanism of the developed microfluidic platform is based on the increase of the overall conductivity with the increase of the concentration of AA, resulting in the decrease of the resistive parameters and increase of the capacitive parameters of the proposed equivalent electrical circuit. The addition of graphene was found to improve the response linearity by $5.28 \%$ and lower the limit of detection and quantification by $12 \%$, compared to the reference structure without graphene.
\end{abstract}

Index Terms- microfluidics, graphene, ascorbic acid, impedance spectroscopy

\section{Introduction}

$\mathrm{N}$ UTRIONAL supplements have been increasingly popular in recent decades. The emerging field of „nutraceuticals“ [1] emphasizes the importance of nutrition and dietary supplements, not only as an essential factor for prevention of diseases and maintaining good health but also as a way to treat various pathological conditions - alone or in combination with drugs. Ascorbic acid (AA) is a natural organic acid that is widely present in fruits. The AA content is also considered as an indicator of the freshness of the fruits. Since the human body does not produce AA, it is essential to have adequate AA intake through the food, drugs or dietary supplements (recommended dietary allowance is $\sim 120 \mathrm{mg} /$ day). AA can be added in pharmaceutical formulations in order to prevent or cure some diseases, e.g., common cold and hypohemia [2].

The conventional techniques for the detection of AA are chromatography [3], spectrophotometry [4], and fluorometry [5]. It has been demonstrated that the liquid chromatography can also be coupled with electrochemical methods to determine the amount of AA in real samples such as oranges and apples, pharmaceutical preparations, and human blood serum [6]. Some recent approaches for AA detection are based on optical fiber sensors [7], [8]. A Germanium-doped photosensitive optical fiber demonstrated a wide linear range of detection from $1 \mu \mathrm{M}$ to $1 \mathrm{mM}$ [7]. Moreover, localized surface plasmon resonance

The results presented in this article received funding from the EU $\mathrm{H} 2020$ research and innovation programme under the Marie Skłodowska-Curie grant agreement no. 872370 - SALSETH, EU H2020 Graphene Flagship Core 3 Grant No. 881603.

Goran M. Stojanović, Tijana Kojić and Mitar Simić are with Faculty of Technical Sciences, University of Novi Sad, 21000 Novi Sad, Serbia (e-mails: sgoran@uns.ac.rs, kojictijana@uns.ac.rs, mitar.simic@uns.ac.rs). based AA sensor exhibited good chemical and mechanical behavior [8]. Despite the fact that optical-based approaches are promising in terms of the sensitivity and reliability in AA detection, above mentioned methods require costly instrumentation such as fusion splicer and spectrophotometer, trained personnel, and time-consuming procedure for sample preparation, which make them not-optimized for in-situ applications outside the laboratory.

Electrical impedance spectroscopy (EIS) is very popular method for describing electrode-solution interfaces and processes on electrodes immersed in solution of the interest. EIS is non-invasive and allows detection of the events in the solution (change of conductivity, permeability or permittivity) that reflects on changes of the measured impedance between electrodes. Therefore, changes in solution can be observed in nondestructive process of the characterization. Additional advantage of EIS is economical aspect as reliable impedance measurement devices can be developed as portable and adopted for specific application in terms of the measurement range. Use of EIS in interface with various sensors was reported by many author, including immunoassay for detection of carcinoembryonic antigen [9]-[11], or dissolved hydrogen sulfide [12], prostate-specific antigen [13], or mercury(II) $\left(\mathrm{Hg}^{2+}\right)$ ion [14], and biosensing of $\mathrm{Cu}^{2+}$ in aqueous solution [15].

Aleksandra Jovanović-Galović is with Faculty of Pharmacy, University of Novi Sad, Novi Sad, 21000 Novi Sad, Serbia (e-mail: aleksandra.jovanovic@faculty-pharmacy.com).

Bojan Pavlović is with PhytoNet AG company, 21000 Novi Sad, Serbia (e-mail: bpavlovic@phytonet.com).

Amaia Zurutuza is with Graphenea, Avenida de Tolosa 76, 20018 Donostia/San Sebastián, Spain (e-mail: a.zurutuza@graphenea.com)

Luca Anzi, Roman Sordan are with L-NESS, Department of Physics, Politecnico di Milano, Via Anzani 42, 22100 Como, Italy (e-mails: luca.anzi@polimi.it, roman.sordan@polimi.it). 
Recently, the application of microfluidic technologies in detection systems become very attractive choice for the manufacturing of sensor platforms [16]. Main features of microfluidic platforms are small size, request for a very small quantity of the analyzed sample and ability to perform multistep analysis with non-turbulent flow of fluids [16]. Moreover, microfluidic technology offers integration of various components in a single platform [16]. Therefore, flexibility, rapid analysis, low fabrication costs, ease of implementation and disposability promote microfluidic technology to a very popular solution in biochemical sensing [17-36]. AA is soluble in water, allowing the application of food microfluidics. Polydimethylsiloxane-based microfluidic chips/sensors have been coupled with ultraviolet-visible (UV/vis) spectroscopy for the quantification of AA [17], [18]. A microfluidic platform based on screen-printed carbon electrodes for the detection of dopamine and AA using chronoamperometric method has been reported [19]. In addition, microfluidic chips can be based on paper, as an inexpensive substrate material, for the realization of analytical devices with good performances. Testing of a microfluidic paper-based prototype for detection and separation of AA and uric acids has been demonstrated [20]. The concentration of AA has been determined in the commercial tablets of $\mathrm{C}$ vitamin by means of a device based on paper and graphite pencil [21]. An electrochemical sensor based on flexible graphite paper for determination of AA, dopamine and uric acid has been fabricated [22].

With the fast development of nanotechnology and discovery of new nanomaterials, such as carbon nanotubes (CNTs) and graphene, new sensing devices were realized for prostatespecific antigen [23], visual screening of $\mathrm{H}_{2} \mathrm{~S}$ [24], gas biosensors [25] and AA detection [26]. CNTs, with their excellent electrical and mechanical properties, have been used as a sensor for AA [27] or for improving AA detection by the electrochemical methods [28]. Thanks to its exceptional electronic properties and two-dimensional structure, graphene has also been used as a building block in many biosensing applications. The graphene/CuPc/PANI nanocomposite structure has been manufactured by electrolytic exfoliation technique for the determination of AA and investigation of its electrochemical characteristics [29], [30]. The complete measurement system based on the complex structure of the magnetic beads-ascorbate oxidase/graphene oxide/indium gallium zinc oxide/aluminium and the microfluidic device has been developed and tested as a biosensor of AA [31]. An AA sensor validated by means of a commercial vitamin $\mathrm{C}$ supplement, applying a structure based on pyrolysed photoresist films and graphene nano-sheets, has been demonstrated [32]. The graphene doped carbon paste electrode has been employed for ensuring good electrochemical current responses for the electrocatalytic oxidation of AA [33]. The simultaneous determination of AA, dopamine, and uric acid has been demonstrated, applying a sensor realized from pristine graphene [34]. The same application has been reported using a nanocomposite of graphene and size-selected Pt nanoparticles [35]. A sensor based on $\mathrm{NiO}$ nanoparticles and graphene composite film for the determination of AA in the presence of folic acid has been demonstrated [36].

Therefore, there is a need for microfluidic sensing devices for rapid separation and determination of dietary supplements. Particularly, it is of great importance to have an accurate method and device for AA detection. Considering that commercially available vitamin $\mathrm{C}$ supplements also contain a significant amount of sweeteners, it is crucial to differentiate AA from isomalt, a widely applied a sugar substitute. Therefore, combination of EIS, microfluidic technologies and graphene is very interesting approach for AA detection and observation if Isomalt is presented.

Here, we present a microfluidic platform for the efficient detection of AA by measuring electrical parameters between $\mathrm{Ag}$ electrodes. The electrodes were inkjet printed with Ag ink to minimize the electrical resistance. Realization of the platform with polyvinylchloride (PVC) thermosensitive foils provided robustness of the platform. The proposed platform uses a chemically unmodified graphene layer in the gap between the Ag electrodes ensuring a cost-effective and straightforward fabrication process. We used graphene as it was reported earlier that graphene enhances sensitivity of the impedance-based sensing platform even with small active surfaces [23-25]. Graphene is excellent candidate for biochemical sensing as it has very good electronic properties, such as high electrical conductivity $\left(3000 \mathrm{~W} \mathrm{~m}^{-1} \mathrm{~K}\right)$, high surface-to-volume ratio $\left(2600 \mathrm{~m}^{2} \mathrm{~g}^{-1}\right)$, high electron transfer rate $\left(15000 \mathrm{~cm}^{2} \mathrm{~V}^{-1} \mathrm{~s}^{-1}\right)$ and exceptional thermal stability [25]. With economical impedance measurements and fitting the electrical response of the sensor to the proposed equivalent electrical circuit, the microfluidic platform enabled a quick determination of the AA concentration in the food supplements tablets.

\section{MATERIALS AND METHODS}

\section{A. Design of microfluidic platform and materials}

The proposed microfluidic platform was fabricated as a multilayered structure composed of PVC transparent foils, as shown in Fig. 1(a). On the bottom PVC foil, which has the role of a substrate, the three pairs of $\mathrm{Ag}$ electrodes were inkjet printed, ensuring the reliability of the entire device. The reasons behind the usage of Ag-electrode are manifold: (a) Ag has the highest electrical conductivity among the conventional conductive materials, (b) Ag-ink is the most often use in combination with Dimatix ink-jet printer DMP-3000, applied in this study, (c) Ag-electrodes provide terminals for connection of this components with external electronics, and (d) graphene layer has an affinity to create a good electrical contact with Agelectrodes. The lateral dimensions of the electrodes were 10 $\mathrm{mm} \times 4 \mathrm{~mm}$, and their thickness was $\sim 250 \mathrm{~nm}$. There is a short gap (length $\sim 0.3 \mathrm{~mm}$ ) between the electrodes (Fig. 1(b)). Graphene was transferred by a wet process on top of the $\mathrm{Ag}$ electrodes, providing an electrical connection between the electrodes. TEM image of the used graphene is shown in Fig. 2. In the middle layer, microfluidic channel was curved using a cutting plotter. At the top layer, PVC foil was used, as a costeffective material, with an inlet and outlet for the tested fluid. The three PVC layers were laminated together at $130{ }^{\circ} \mathrm{C}$ to 
obtain a compact portable microfluidic platform, which is easy to handle and manipulate. The fabricated microfluidic chip is depicted in Fig. 1(b). The overall chip dimensions were $2.5 \mathrm{~cm}$ $\times 5.5 \mathrm{~cm}$.

(a)
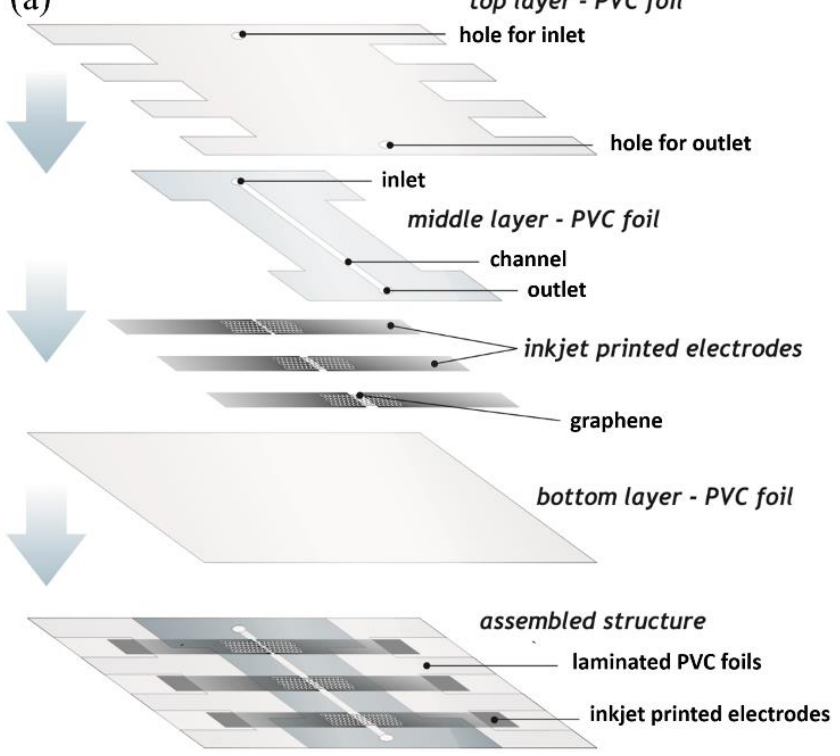

(b)

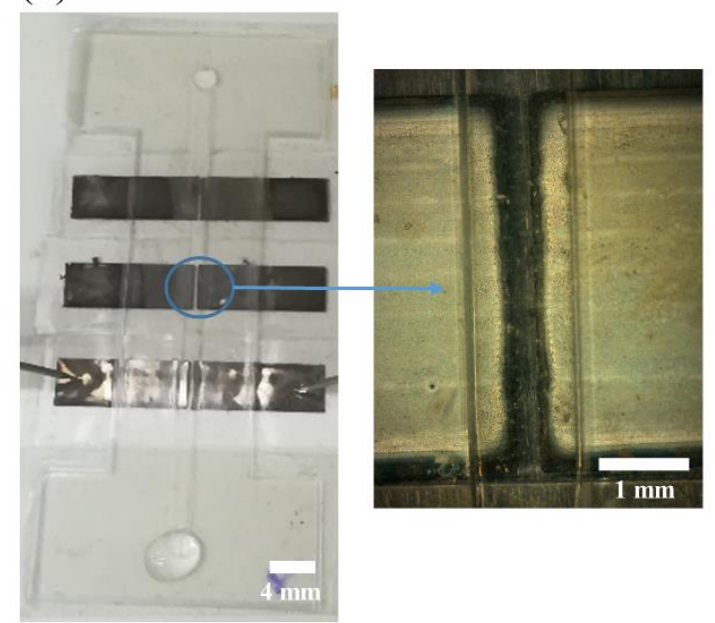

Fig. 1. Design of the microfluidic platform, (b) A photo of the fabricated microfluidic platform and a zoomed-in part of the gap between the electrodes.

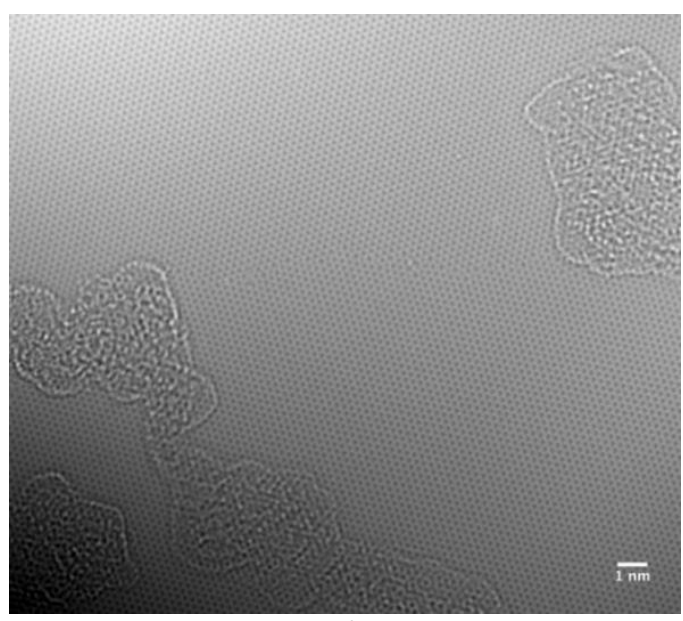

Fig. 2. TEM image of the used graphene.

\section{B. Fabrication and characterization methods}

The deposition material printer DMP-3000 (Dimatix) was used to print electrodes on PVC foil $\left(80 \mu \mathrm{m}\right.$ thick, MBL ${ }^{\circledR}$ 80MIC A4 hot lamination foil, Minoan Binding Laminating d.o.o. Serbia). Inkjet printing parameters were: the minimum droplet diameter was $36 \mu \mathrm{m}$ and the spacing between drops was $18 \mu \mathrm{m}$. The ink-jet printing process, using Dimatix printer, was optimized in order to achieve a stable stream of droplets from cartridge towards the surface, without the so-called "satellite" drops that are formed after basic drops and that cause shorting of printed electrodes and "tail" drops which usually leads to a "messy" and unsuccessful printing structure. The quality of the printed continuous conductive lines depends on the droplet diameter and spacing between drops and they actually determine the overlapping degree of the droplets. These two parameters were optimized based on our previous results [37], [38], in order to have conductive line with good conductivity and flat edges of the line. If droplet diameter will be greater than $36 \mu \mathrm{m}$, the wave edges of the lines will be obtained. If the spacing will be greater than $18 \mu \mathrm{m}$, there is a possibility to have gaps in the conductive lines. Graphene was wet-transferred in the gap between the $\mathrm{Ag}$ electrodes (printed using $\mathrm{Ag}$ nanoparticle ink). Microfluidic channel was created by a xurography technique. Namely, the cutting plotter (CE6000-60 PLUS, Graphtec America, Inc., USA) was used for engraving straight line (with a width of $1 \mathrm{~mm}$ and length of $4 \mathrm{~cm}$ ) and holes (diameter of $2 \mathrm{~mm}$ ) in the PVC foil, for the inlet and outlet. For engraving, a cutting blade with a $45^{\circ}$ angled tip was used. A hot laminator was used to laminate the individual layers into a single compact structure. Electrochemical measurements were performed using PalmSens4 instrument at room temperature. The PalmSens4 was a computer-controlled via a Bluetooth link, and software tool PS Trace 5.8 was used for performing measurements and exporting the measured data.

\section{Preparation of the testing samples}

Tablets were prepared by PhytoNet AG [39], in accordance with good manufacturing practice. The first group of tablets comprised pure isomalt which is water-soluble and cannot be bound on the active substance (AA in this case). The second group of tablets comprised pure AA. The third group of tablets comprised a mixture of $50 \%$ of isomalt and $50 \%$ of AA. For the electrochemical measurements, the tablets were dissolved in 5 $\mathrm{ml}$ of double distilled water ( $\mathrm{ddH} 2 \mathrm{O}$ ), and filtered through a $0.22 \mu \mathrm{m}$ syringe filter ensuring bacteria-free samples. The procedure undertaken in the preparation of the sample in our work, is known as sterilization by filtration. Due to the pore size of the applied filters $(0.22 \mu \mathrm{m})$, all particles greater than 0.22 $\mu \mathrm{m}$ will be trapped by filter. This include bacteria [40], [41]. Each step was performed in sterile tubes and syringes and handled using sterile gloves. The microfluidic channel was cleaned with deionized water after each measurement.

\section{RESULTS AND DISCUSSION}

\section{A. Electrochemical impedance spectroscopic analysis}

We implemented the impedance spectroscopy analysis to characterize the samples without and with graphene between the Ag electrodes. The PalmSens4 instrument was setup in twoelectrode configuration, and frequency range from $1 \mathrm{~Hz}$ to 100 
$\mathrm{kHz}$ was covered with 50 logarithmically spaced points. Measured impedance magnitude (or "impedance" for short) as a function of frequency is shown in Fig. 3 .
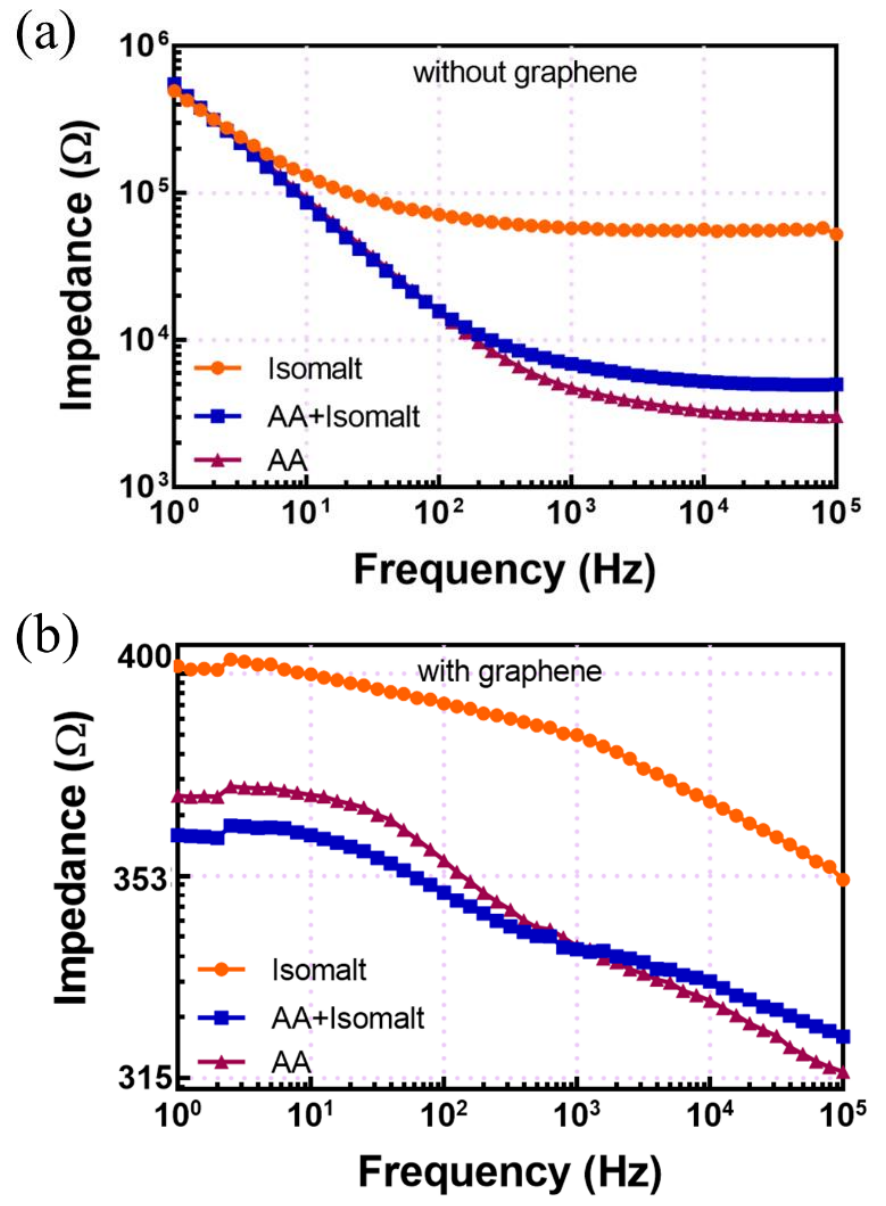

Fig. 3. Impedance as a function of frequency for a sample (a) without graphene and (b) with graphene.

In the structure without graphene, the smallest impedance was obtained with pure AA inside the channel. A mixture of 50 $\% \mathrm{AA}+50 \%$ isomalt had larger impedance, while the largest impedance was obtained with pure isomalt. The measured impedance was in order of tens/hundreds of $\mathrm{k} \Omega$ which was dominated by the low conductivity medium $\left(\mathrm{ddH}_{2} \mathrm{O}\right)$ between the electrodes. In general, pure $\mathrm{ddH}_{2} \mathrm{O}$ is very similar to an insulator, but it has some low finite conductivity. Such low conductivity medium can be modelled by a series RC network [42]. Values of the network elements mostly depend on ion concentration, mobility, and size of the particles of active substance. A typical plot of the electrical impedance of the series RC network versus frequency (with logarithmic scale on both axes) is linear at low frequencies and has a plateau at high frequencies [42]. It exhibits a very similar trend as obtained in Fig.3(a).

An addition of a graphene layer, in the gap between the Ag electrodes of the microfluidic platform, did not change the relative order of the impedances: the structure with AA inside the channel had the lowest impedance, while the structure with isomalt had the highest impedance (Fig. 3(b)). However, the addition of graphene resulted in a significant decrease of the impedance compared to the microfluidic platform without graphene. For example, at a frequency of $10 \mathrm{kHz}$, the impedance without graphene for isomalt, $\mathrm{AA}+$ isomalt, and $\mathrm{AA}$ was $56.6 \mathrm{k} \Omega, 5.22 \mathrm{k} \Omega$, and $3.31 \mathrm{k} \Omega$, respectively. At the same frequency, the measured impedance with graphene was 368.6 $\Omega, 332.8 \Omega$ and $329.2 \Omega$, for isomalt, AA + isomalt and AA, respectively. The advantages of the lower impedance of the microfluidic platform with graphene are easier electron transfer and interfacing with a measurement unit.

Fig. 4 shows calibration curves for chips without and with graphene at the frequency of $10 \mathrm{kHz}$. The graphs also depict the corresponding linear fits.

(a)

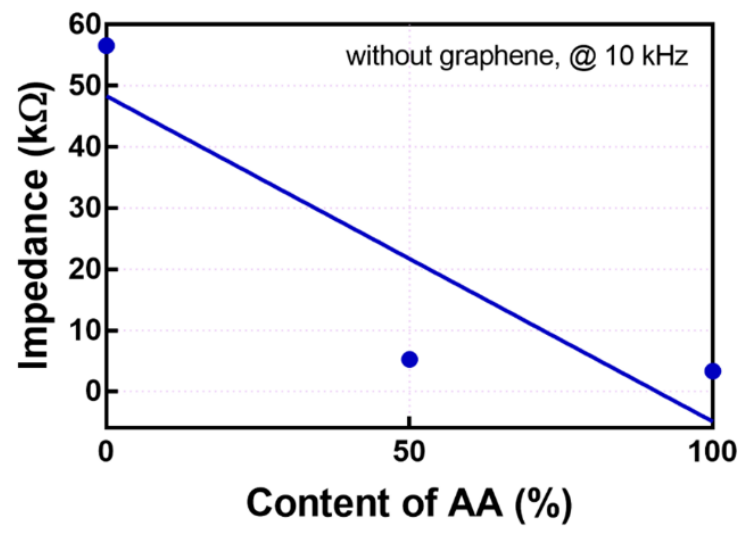

(b)

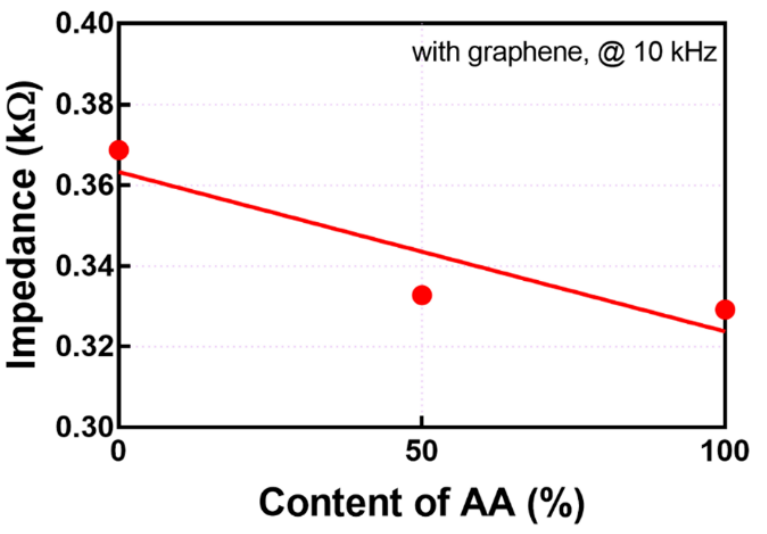

Fig. 4. Calibration curves for chips: (a) without graphene, (b) with graphene. The impedances were measured at a frequency of $10 \mathrm{kHz}$.

In order to quantitatively compare performances of the studied microfluidic structures with and without graphene, the coefficient of linearity $\left(\mathrm{R}^{2}\right)$, limit of detection (LOD), and limit of quantification (LOQ) were calculated. The LOD (Table I) was calculated as $3.3 \cdot S t e / b$ and LOQ as $10 \cdot S t e / b$, where Ste is the standard error from regression statistics and $b$ is the slope coefficient of the calibration curve of the linear regression lines. The confidence interval of $95 \%$ was implemented.

TABLE I

\begin{tabular}{lccc}
\multicolumn{4}{c}{ QuANTITATIVE STUDY OF SENSING PERFORMANCES } \\
& $\mathrm{R}^{2}$ & $|\mathrm{LOD}|$ & $|\mathrm{LOQ}|$ \\
\hline Without graphene & 0.777 & 125.01 & 378.82 \\
\hline With graphene & 0.818 & 110.03 & 333.43 \\
\hline
\end{tabular}

It can be seen in Table I that chip with graphene has a better 
linearity for $5.2 \%$, and lower LOD and LOQ for $12 \%$ in comparison to the structure without graphene. Therefore, in addition to the excellent electrical properties of graphene in terms of high electrical conductivity, high surface-to-volume ratio, high electron transfer rate and exceptional thermal stability [25], addition of graphene improved linearity and enabled lower levels of detection and quantification. Obtained characteristics are the additional arguments for incorporating the graphene layer in our microfluidic platform.

The reproducibility of the proposed sensing platform was evaluated by fabricating three individual pair of silver electrodes on the same substrate, which were implemented for conducting the same experimental procedure. A satisfactory relative standard deviation (RSD) of $4.17 \%$ was obtained. The presented platform is very convenient with regard to reusability, because it is necessary to rinse the microfluidic channel with distilled water and after that to enter new solution intended for analysis and the measurement procedure can be repeated. Using one pair of electrode, it is possible to perform many consecutive measurements due to excellent mechanical properties of graphene layer and thanks to the fact that it is not necessary to apply any high pressure on injected solution in the microfluidic channel.

A significantly smaller impedance of the platform with graphene was attributed to the high conductivity of graphene (1738 S/m, equivalent to a resistivity of $0.0575 \Omega \mathrm{cm}$ ) [43]. The transferred graphene layer established an additional lowresistance current path between the electrodes. Thus, the resulting structure had two phases: low conductivity $\left(\mathrm{ddH}_{2} \mathrm{O}\right.$ with $\mathrm{AA} /$ isomalt) and high conductivity phase (graphene), as illustrated in Fig. 5(a). Fig. 5(b) depicts the electrical model of the fabricated sensor.
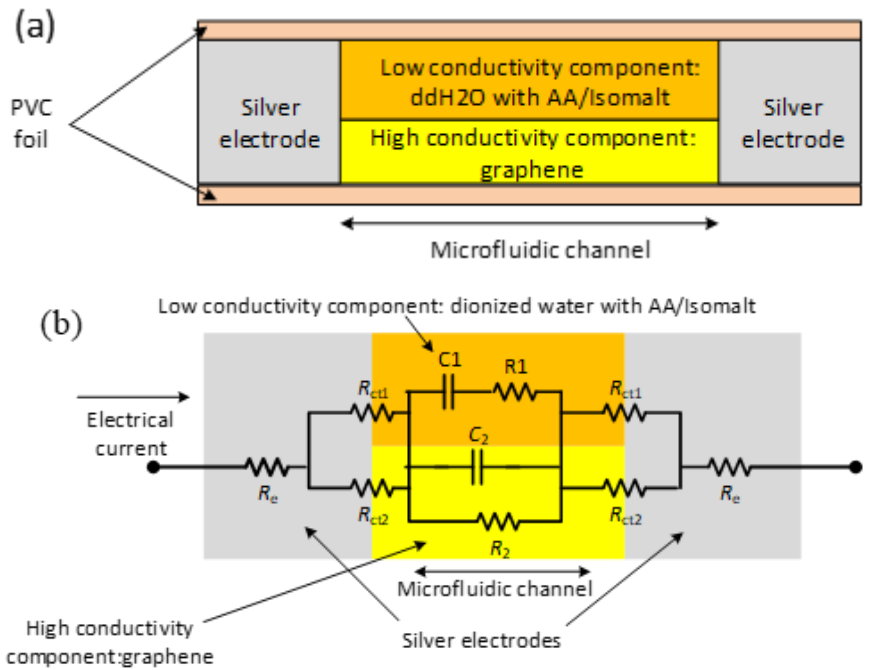

Fig. 5. (a) Resulting structure with low conductivity phase on the top of the graphene layer (not in scale), (b) lumped electrical model of the fabricated sensors.

The low conductivity phase can be modelled as a series connection of a resistor $\left(R_{1}\right)$ and capacitor $\left(C_{1}\right)$, as shown in Fig. 6(a). This can be understood from Fig. 3(a), where impedance change with frequency is presented for the system without graphene. Resistance $R_{1}$ is the solution resistance, while $C_{1}$ models dielectric properties of the solution. The high conductivity phase is composed of graphene layer, and it is modelled as a resistor $\left(R_{2}\right)$ and capacitor $\left(C_{2}\right)$ connected in parallel [44], [45]. $R_{2}$ is the resistance of graphene, while $C_{2}$ is the interface capacitance due to the existence of the interlayer between the metal electrodes and graphene. Parallel connection of the impedances of the low and high conductivity phases is connected in series with the resistance of the metal electrodes and connecting wires to the measurement device $\left(R_{\mathrm{e}}\right)$, as well as charge transfer resistances between the electrodes and low/high conductivity mediums ( $R_{\mathrm{ct} 1}$ and $R_{\mathrm{ct} 2}$, respectively). The electrical circuit shown in Fig. 5(b) is equivalent to the simplified circuit shown in Fig. 6(a), where the series resistance $R_{\mathrm{S}}=2\left(R_{\mathrm{e}}+R_{\mathrm{ct} 1} R_{\mathrm{ct} 2} /\left(R_{\mathrm{ct} 1}+R_{\mathrm{ct} 2}\right)\right)$. The Nyquist plot of the circuit impedance is shown in Fig. 6(b) and it exhibits two semicircles, as theoretically expected. The plot of the same impedance versus frequency is shown in Fig. 6(c). The measured Fig. 3(b) is very similar to Fig. 6(c).
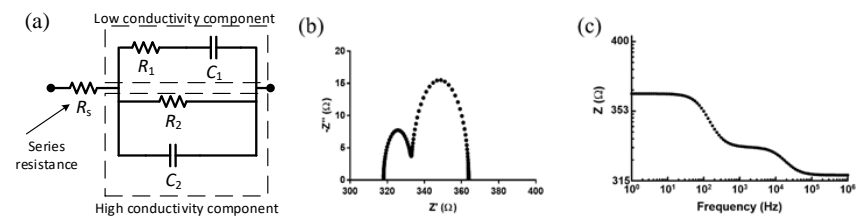

Fig. 6. (a) Proposed equivalent circuit. (b) Typical Nyquist plot of a proposed equivalent electrical circuit. Z' and Z" are the real and imaginary part of the complex impedance. (c) Corresponding impedance plot versus frequency (logarithmic scale on both axes).

Fig. 7(a) shows Nyquist plots of the measured impedances of the platform without graphene. However, the Nyquist plots for AA (blue line) and $50 \% \mathrm{AA}+50 \%$ isomalt (dark magenta) intersect and overlap at low and moderate frequencies making distinction between these two cases difficult. This suggests that higher operating frequencies are required, which can be expensive for in-situ applications.

Fig. 7(b) shows the Nyquist plots of the measured impedances of the platform with graphene. The presence of two semicircles suggests the existence of two materials with different electrical properties which confirms the discussed model.

We used MEISP software tool to fit measured values, and estimated values of the model parameters are shown in Table II.

TABLE II

ESTIMATED VALUES OF PARAMETERS OF THE EQUIVALENT ELECTRICAL

\begin{tabular}{lccccc}
\multicolumn{7}{c}{ CIRCUIT SHOWN IN FIGURE 4(A). } \\
Fluid & $R_{\mathrm{s}}(\Omega)$ & $R_{1}(\Omega)$ & $C_{1}(\mu \mathrm{F})$ & $R_{2}(\Omega)$ & $C_{2}(\mu \mathrm{F})$ \\
\hline Isomalt & 362.92 & 55.15 & 26.49 & 33.37 & 1.47 \\
\hline AA+Isomalt & 330.21 & 27.89 & 41.17 & 31.70 & 4.10 \\
\hline AA & 326.91 & 27.61 & 41.59 & 31.38 & 4.14 \\
\hline
\end{tabular}

The quality of the proposed model was estimated by calculating root-mean-square errors (RMSEs) for the real and imaginary part of the complex impedance of the microfluidic platform. Table III shows that RMSEs of the real and imaginary parts of the impedance are $<10 \Omega$, which is satisfactory considering that the overall order of impedance magnitudes were hundreds of ohms (Fig. 7). 
(a)

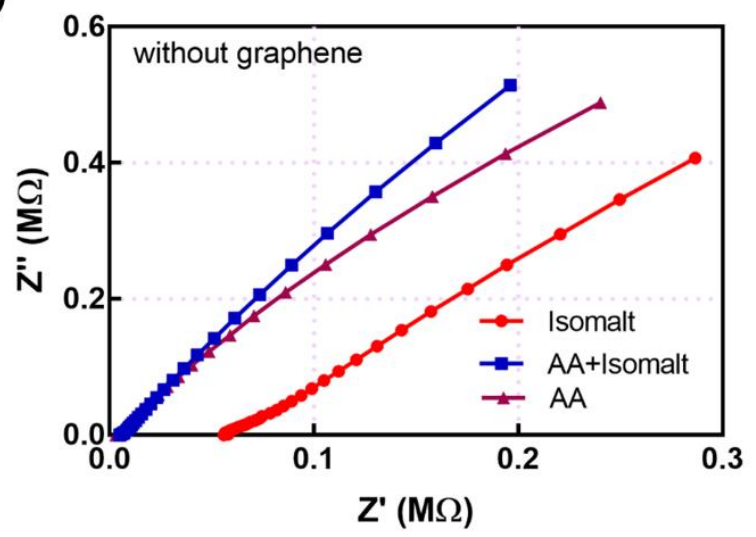

(b)

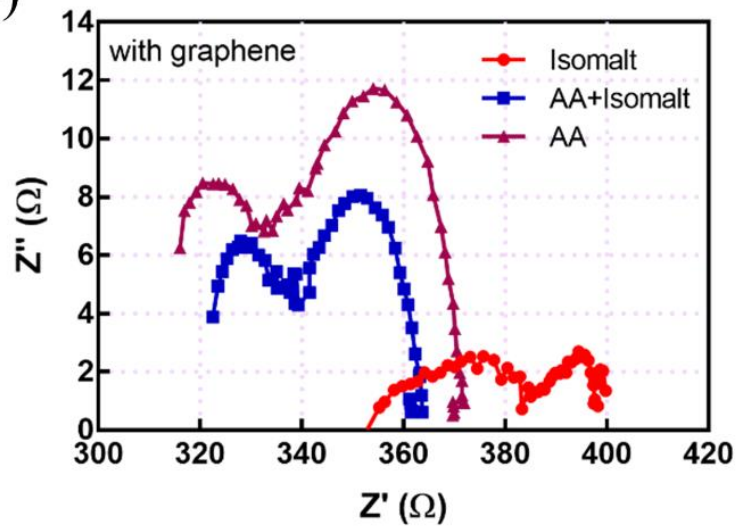

Fig. 7. Nyquist plots for the platform (a) without graphene and (b) with graphene.

TABLE III

RMSE OF THE REAL AND IMAGINARY PART OF THE IMPEDANCE OF THE MICROFLUIDIC PLATFORM WITH GRAPHENE WHEN ISOMALT, ISOMALT + AA, AND AA ARE APPLIED.

\begin{tabular}{lcc} 
Fluid & $\operatorname{RMSE}_{\mathrm{R}}(\Omega)$ & $\operatorname{RMSE}_{\mathrm{X}}(\Omega)$ \\
\hline Isomalt & 2.74 & 3.70 \\
\hline AA+Isomalt & 2.39 & 2.23 \\
\hline AA & 8.63 & 3.74 \\
\hline
\end{tabular}

\section{B. Discussion and description of the sensing} mechanism

Isomalt $\left(\mathrm{C}_{12} \mathrm{H}_{24} \mathrm{O}_{11}\right)$ is a mixture of two sugar alcohols: gluco-mannitol and gluco-sorbitol. It has relatively low solubility in water at low temperatures $\left(25 \mathrm{~g} / 100 \mathrm{~g}\right.$ at $\left.25^{\circ} \mathrm{C}\right)$ with a high tendency to crystallize [46]. However, $\mathrm{AA}\left(\mathrm{C}_{6} \mathrm{H}_{8} \mathrm{O}_{6}\right)$ is a polar organic molecule that has high water solubility $(290 \mathrm{~g} / \mathrm{L}$ at $20^{\circ} \mathrm{C}$ ) [47], forming more homogenous structure. AA has 4 hydroxyl groups in its structure providing hydrogen bonds with water molecules and leading to very fast equilibrium and acid nature of the resulting solution after dissolution [48]. The acid nature of $\mathrm{AA}$ is recognized from its $\mathrm{pH}$ value, which is between 2.4 and 2.8 [49].

Acids ionize (dissociate) in solution, increasing the number of the ions that carry the electric charge, and therefore their presence increases the solution conductivity [50]. From Table II, it can be seen that $R_{1}$ is lower for AA in comparison to Isomalt. Moreover, the addition of $\mathrm{AA}$ to isomalt reduces $R_{1}$ compared to that of pure isomalt. However, $C_{1}$ increased (due to the increase of dielectric constant stemming from the reduction of the electric field in the conducting medium) as the concentration of AA is increased. Table II supports this conclusion.

As it was expected, resistance of the graphene layer $\left(R_{2}\right)$ was changed just a little bit from $33.37 \Omega$ (isomalt) to $31.38 \Omega$ (AA). Rather than being a constant, resistance of the graphene layer slightly decreased due to interaction with AA. Moreover, as the conductivity of graphene is increased, the decrease of insulating properties of the device leads to the increase of $C_{2}$. Table II supports these conclusions, providing additional verification of the presented sensing mechanism and proposed equivalent electrical circuit.

Moreover, it can be noted that series resistance $R_{\mathrm{S}}$ decreases with increased AA concentration. Decrease of $R_{\mathrm{s}}$ provides a main justification for adding graphene. As noted above, $R_{\mathrm{S}}$ is composed of a series connection of the electrode resistances and connecting wires $\left(R_{\mathrm{e}}\right.$, which should not change with the concentration of AA) and equivalent resistance of the parallel connection of the charge transfer resistances $\left(R_{\mathrm{ct} 1}\right.$ and $R_{\mathrm{ct} 2}$ in Fig. 4(b)). As graphene layer provides current path of very high conductivity, it has small charge transfer resistance, which reduces the resistance of the parallel connection of $R_{\mathrm{ct} 1}$ and $R_{\mathrm{ct} 2}$. Therefore, the addition of graphene layer enhances electron transfer [51].

The sensing mechanism of the developed microfluidic platform is based on the increase of the overall conductivity with the increase of the concentration of AA, resulting in the decrease of the resistive parameters and increase of the capacitive parameters of the equivalent electrical circuit. The proposed model better fits (lower RMSE values in Table III) the impedance of the microfluidic platform in the presence of isomalt.

\section{Performance comparison of the proposed device with existing ones}

Prior to the conclusion section where we will emphasize our main results, we have analysed our approach and relevant similar works.

As it can be seen in Table IV, many groups were focused on the development of the platforms for precise detection of the various AA concentration, Therefore, of great importance is to have an accurate method and device for AA detection. Considering that commercially available vitamin $\mathrm{C}$ supplements also contain a significant amount of sweeteners, it is also crucial to differentiate AA from isomalt, a widely applied a sugar substitute. However, as it can be seen from Table I there is a lack of such studies and our study covers exactly that gap. 
TABLE IV

COMPARISON OF RELATED WORKS AA SENSING

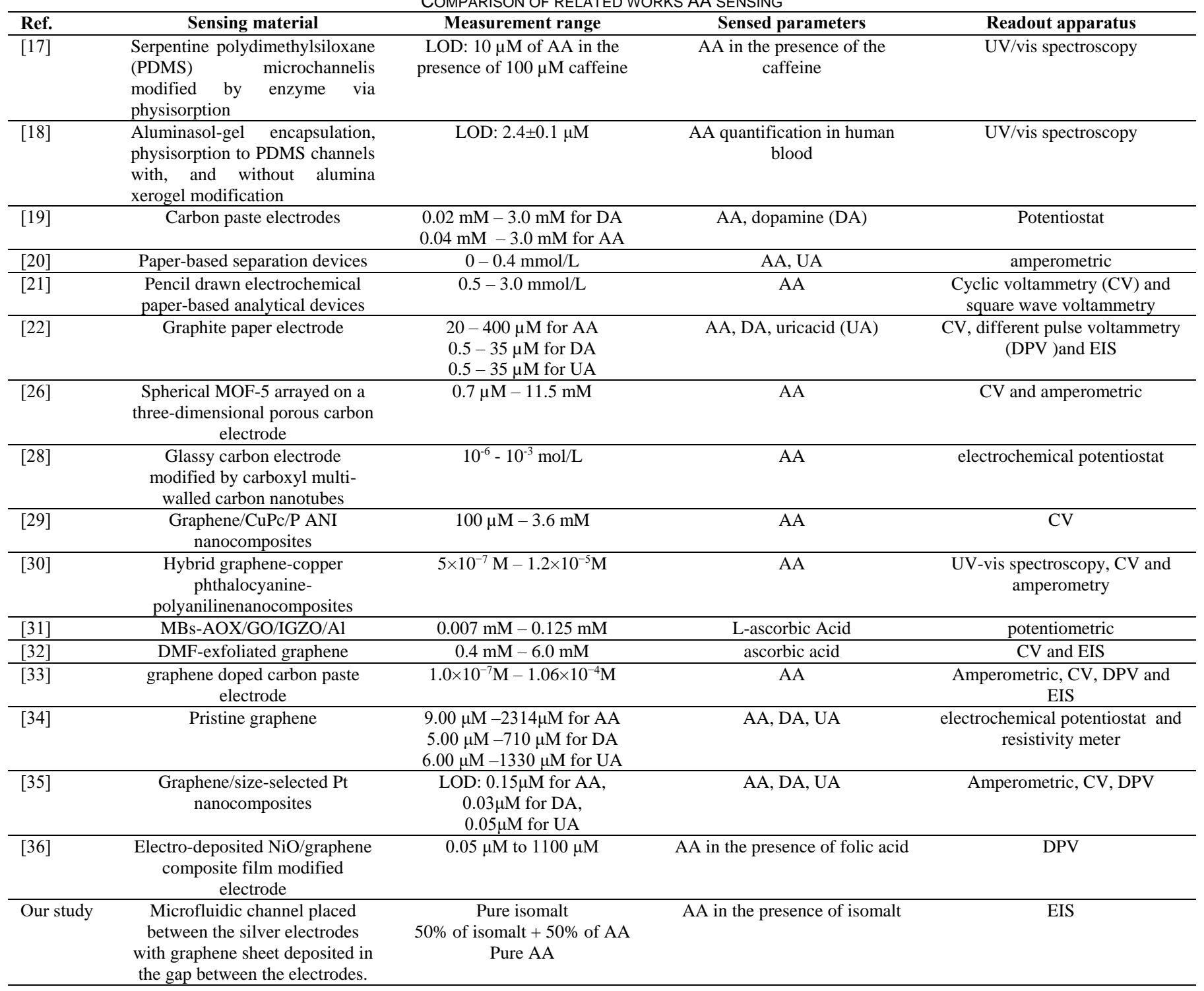

\section{CONCLUSION}

In this study, we presented a compact and robust microfluidic platform for the efficient detection of AA. The proposed platform can be used in the food and dietary supplement industry as well as for authenticity checking of-the-shelf products. Such microfluidic platform, which is based on the measurement of electrical impedance, enables determination of the concentration of supplements, and could be of a significant benefit both from the health and economic standpoint. The availability of the proposed microfluidic device would help the interested parties: consumers - to choose the desired product, pharmacists - to sell only high-quality supplements, and producers - to find the right formulations that enable prolonged shelf-life and save by not having to add more than necessary of the active substance into the product.

We provided details regarding design, fabrication and characterization of the microfluidic platform suitable for the detection of AA. The equivalent electrical circuit, based on the theoretical analysis and experimental measurements, was verified. The sensing mechanism and the electrical parameters in the presence of AA in the microfluidic channel were described. We found that our microfluidic platform is capable of differentiating between the solutions comprising pure AA, mixture of AA and isomalt, and pure isomalt.

The future work should be directed to the development and implementation of the device for in-situ impedance measurement with the presented microfluidic platform. Such an approach should reduce the overall cost of the system and processing time from taking a sample to providing information on ascorbic acid presence. Moreover, determination of serum Ascorbic acid concentration is frequently performed in Medical biochemistry laboratories, usually as part of a set of automated biochemical analyses. A separate set of experiments will be designed in order to validate the use of our sensor in the clinical setting, discover potential interference substances otherwise 
present in the biological materials, and ultimately determine reproducibility and other performance characteristics (i.e. cutoff values) of the innovative method. In the course of sensor validation ASA in serum, plasma, erhytrocyte hemolisate, and urine will be included. Other biological materials, such as saliva or sweat samples are also taken into consideration.

\section{REFERENCES}

[1] A. Santini and E. Novellino, "Nutraceuticals - shedding light on the grey area between pharmaceuticals and food", Expert Review of Clinical Pharmacology, vol. 11, no. 6, pp. 545-547, 2018, doi:10.1080/17512433.2018.1464911.

[2] J. C. Rickman, D. M. Barrett and C. M. Bruhn, "Nutritional comparison of fresh, frozen and canned fruits and vegetables. Part 1. Vitamins C and B and phenolic compounds", J Sci Food Agric, vol. 87, pp. 930-944, 2007.

[3] I. Klimczak and A. Gliszczynskaswiglo, "Comparison of MPLC and HPLC methods for determination of vitamin C", Food Chem, vol. 175, pp. 100-105, 2015.

[4] R.O. Hassan and A. T. Faizullah, "Reverse-FIA with Spectrophotometric Detection Method for Determination of Vitamin C", J. Iran. Chem. Soc. Vol. 8, pp. 662-673, 2011.

[5] T. Maki, N. Soh, K. Nakano and T. Imato, "Flow injection fluorometric determination of ascorbic acid using perylenebisimide-linked nitroxide", Talanta, vol. 85, pp. 1730-1733, 2011.

[6] Z. Gazdik, O. Zitka, J. Petrlova, V. Adam, J. Zehnalek, Ales Horna, Vojtech Reznicek, Miroslava Beklova and Rene Kizek, "Determination of Vitamin C (Ascorbic Acid) Using High Performance Liquid Chromatography Coupled with Electrochemical Detection", Sensors, vol. 8, pp. 7097-7112, 2008, doi: 10.3390/s8117097.

[7] S. Kumar, R. Singh, Q. Yang, S. Cheng, B. Zhang and B. K. Kaushik, "Highly Sensitive, Selective and Portable Sensor Probe Using Germanium-Doped Photosensitive Optical Fiber for Ascorbic Acid Detection," IEEE Sensors Journal, vol. 21, no. 1, pp. 62-70, 2021, doi: 10.1109/JSEN.2020.2973579.

[8] G. Zhu, N. Agrawal, R. Singh, S. Kumar, B. Zhang, C. Saha, C. Kumar, "A novel periodically tapered structure-based gold nanoparticles and graphene oxide-Immobilized optical fiber sensor to detect ascorbic acid", Optics \& Laser Technology, vol. 127, p. 106156, 2020.

[9] L. Hou, Y. Cui, M. Xu, Z. Gao, J. Huang J and D. Tang, "Graphene oxidelabeled sandwich-type impedimetric immunoassay with sensitive enhancement based on enzymatic 4-chloro-1-naphthol oxidation", Biosensors and Bioelectronics, vol. 47, pp. 149-56, 2013.

[10] L. Hou, Y. Tang, M. Xu, Z. Gao and D. Tang, "Tyramine-based enzymatic conjugate repeats for ultrasensitive immunoassay accompanying tyramine signal amplification with enzymatic biocatalytic precipitation", Analytical chemistry, vol. 86, no. 16, pp.8352-8358, 2014.

[11] L. Hou, X. Wu, G. Chen, H. Yang, M. Lu and D. Tang, "HCR-stimulated formation of DNAzyme concatamers on gold nanoparticle for ultrasensitive impedimetric immunoassay", Biosensors and Bioelectronics, vol. 68, pp. 487-493, 2015.

[12] J. Zhuang, L. Fu, W. Lai, D. Tang and G. Chen, "Target-stimulated metallic $\mathrm{HgS}$ nanostructures on a DNA-based polyion complex membrane for highly efficient impedimetric detection of dissolved hydrogen sulfide", Chemical Communications, vol. 49, no. 95, pp. 11200$11202,2013$.

[13] L. Hou, Z. Gao, M. Xu, X. Cao, X. Wu, G. Chen and D. Tang, “ DNAzyme-functionalized gold-palladium hybrid nanostructures for triple signal amplification of impedimetric immunosensor", Biosensors and Bioelectronics, vol. 54, pp. 365-371, 2014.

[14] Z. Qiu, D. Tang, J. Shu, G. Chen and D. Tang, "Enzyme-triggered formation of enzyme-tyramine concatamers on nanogold-functionalized dendrimer for impedimetric detection of $\mathrm{Hg}$ (II) with sensitivity enhancement", Biosensors and Bioelectronics, vol. 75, pp.108-115, 2016.

[15] M. Xu, Z. Gao, Q. Wei, G. Chen and D. Tang, "Hemin/G-quadruplexbased DNAzyme concatamers for in situ amplified impedimetric sensing of copper (II) ion coupling with DNAzyme-catalyzed precipitation strategy", Biosensors and Bioelectronics, vol. 74, pp. 1-7, 2015.

[16] N. Nesakumar, S. Kesavan, C.-Z. Li and S. Alwarappan, "Microfluidic Electrochemical Devices for Biosensing", Journal of Analysis and Testing, vol. 3, pp. 3-18, 2013, doi: 10.1007/s41664-019-0083-y
[17] H. Bi, A. C. Fernandes, S. Cardoso and P. Freitas, "Interference-blind microfluidic sensor for ascorbic acid determination by UV/vis spectroscopy", Sensors and Actuators B, vol. 224, pp. 668-675, 2016, doi: 10.1016/j.snb.2015.10.072

[18] H. Bi, C. M. Duarte, M. Brito, V. Vilas-Boas, S. Cardoso and P. Freitas, "Performance enhanced UV/vis spectroscopic microfluidic sensor for ascorbic acid quantification in human blood", Biosensors and Bioelectronics, vol. 85, pp. 568-572, 2016, doi: 10.1016/j.bios.2016.05.054

[19] A. Suea-Ngam, P. Rattanarat, O. Chailapakul and M. Srisa-Art, "Electrochemical droplet-based microfluidics using chip-based carbon paste electrodes for high-throughput analysis in pharmaceutical applications", Anal Chim Acta, vol. 883, pp. 45-54, 2015, doi: 10.1016/j.aca.2015.03.008.

[20] R. F. Carvalhal, M. S. Kfouri, M. H. de O. Piazetta, A. L. Gobbi and L. T. Kubota, "Electrochemical Detection in a Paper-Based Separation Device", Anal. Chem, vol. 82, pp. 1162-1165, 2010, doi: 10.1021/ac902647r

[21] V. X. G. Oliveira, A. A. Dias, L. L. Carvalho, T. M. G. Cardoso, F. Colmati, W. K. T. Coltro, "Determination of ascorbic acid in commercial tablets using pencil drawn electrochemical paper-based analytical devices", Analytical sciences, vol. 34, pp. 91-95, 2018, doi: 10.2116/analsci.34.91

[22] W. Cai, T. Lai, H. Du, and J. Ye, "Electrochemical determination of ascorbic acid, dopamine and uric acid based on an exfoliated graphite paper electrode: a high performance flexible sensor", Sens. Actuators B: Chem., vol. 193, pp. 492-500, 2014.

[23] G. Cai, Z. Yu, R. Ren and D. Tang, "Exciton-plasmon interaction between AuNPs/graphene nanohybrids and CdS quantum dots/TiO2 for photoelectrochemical aptasensing of prostate-specific antigen", ACS sensors, vol. 3, no. 3, pp. 632-639, 2018.

[24] J. Shu, Z. Qiu and D. Tang, "Self-referenced smartphone imaging for visual screening of $\mathrm{H} 2 \mathrm{~S}$ using $\mathrm{Cu} \times \mathrm{O}$-polypyrrole conductive aerogel doped with graphene oxide framework", Analytical chemistry, vol. 90, no. 16, pp. 9691-9694, 2018.

[25] R. Zeng, Z. Luo, L. Zhang and D. Tang, "Platinum nanozyme-catalyzed gas generation for pressure-based bioassay using polyaniline nanowiresfunctionalized graphene oxide framework", Analytical chemistry, vol. 90, no. 20, pp. 12299-12306, 2018.

[26] Y. Song, C. Gong, D. Su, Y. Shen, Y. Song and L. Wang, "A novel ascorbic acid electrochemical sensor based on spherical MOF-5 arrayed on a three-dimensional porous carbon electrode", Anal. Methods, vol. 8, pp. 2290, 2016, doi: 10.1039/C6AY00136J

[27] X. Liu, X. Li, Y. Xiong, Q. Huang, X. Y. Li, Y. L. Dong, P. Liu, and Z. C. Zhang, "A glassy carbon electrode modified with the nickel (II)Bis(1,10-phebabthroline) complex and multi-walled carbon nanotubes, and its use as a sensor for ascorbic acid", Microchim. Acta, vol. 180, pp. 1309-1316, 2013, doi: 10.1039/C5AY03271G

[28] B. S. He, J. X. Zhang, "Electrochemical determination of vitamin C on glassy carbon electrode modified by carboxyl multi-walled carbon nanotubes", Int. J. Electrochem. Sci., vol. 10, pp. 9621-9631, 2015.

[29] S. Pakapongpan, J. P. Mensing, T. Lomas and A. Tuantranont, "Electrochemical Sensor for Ascorbic Acid Based on Graphene/CuPc/PANI Nanocomposites", IEEE International Conference on Electron Devices and Solid State Circuit (EDSSC), 3-5 Dec. 2012, doi: 10.1109/EDSSC.2012.6482864.

[30] S. Pakapongpan, J. P. Mensing, D. Phokharatkul, T. Lomas and A. Tuantranont, "Highly selective electrochemical sensor for ascorbic acid based on a novel hybrid graphene-copper phthalocyanine-polyaniline nanocomposites", Electrochimica Acta, vol. 133, pp. 294-301, 2014, doi: 10.1016/j.electacta.2014.03.167

[31] J.-C. Chou, Y.-X. Wu, P.-Y. Kuo, C.-H. Lai, Y.-H. Nien, S.-H. Lin, S.-J. Yan, and C.-Y. Wu, "Determination of L-ascorbic Acid Using MBsAOX/GO/IGZO/Al by Wireless Sensing System and Microfluidic Framework", IEEE Access, vol. 7, pp. 45872-45880, 2017, 10.1109/ACCESS.2019.2907964

[32] G. P. Keeley, A. O’Neill, N. McEvoy, N. Peltekis, J. N. Colemanac and G. S. Duesberg, "Electrochemical ascorbic acid sensor based on DMFexfoliated graphene”, J. Mater. Chem., vol. 20, pp. 7864-7869, 2010, doi: 10.1039/COJM01527J

[33] F. Li, J. Li, Y. Feng, L. Yang, Z. Du, "Electrochemical behavior of graphene doped carbon paste electrode and its application for sensitive determination of ascorbic acid", Sensors and Actuators B, vol. 157, pp. 110-114, 2011, doi: 10.1016/j.snb.2011.03.033 
[34] S. Qi, B. Zhao, H. Tang, X. Jiang, "Determination of ascorbic acid, dopamine, and uric acid by a novel electrochemical sensor based on pristine graphene", Electrochimica Acta, vol. 161, pp. 395-402, 2015, doi: $10.1016 / j$.electacta.2015.02.116

[35] C.-L. Suna, H.-H. Leea, J.-M. Yanga and C.-C. Wu, "The simultaneous electrochemical detection of ascorbic acid, dopamine, and uric acid using graphene/size-selected Pt nanocomposites", Biosensors and Bioelectronics, vol. 26, pp. 3450-3455, 2011, doi: 10.1016/j.bios.2011.01.023

[36] B. Liu, L. Luo, Y. Ding, X. Si, Y. Wei, X. Ouyang, and D. Xu, "Differential pulse voltammetric determination of ascorbic acid in the presence of folic acid at electro-deposited $\mathrm{NiO} /$ graphene composite film modified electrode", Electrochimica Acta, vol. 142, pp. 336-342, 2014, doi: 10.1016/j.electacta.2014.07.126

[37] T. Kojić, G. Stojanović, A. Miletić, M. Radovanović, H. Al-Salami, F. Arduini, "Testing and Characterization of Different Papers as Substrate Material for Printed Electronics and Application in Humidity Sensor", Sensors and Materials, vol. 31, no. 9, pp. 2981-2995, 2019.

[38] D. Vasiljević, A. Mansouri, L. Anzi, R. Sordan, G. Stojanović, "Performance Analysis of Flexible Ink-jet Printed Humidity Sensors Based on Graphene Oxide", IEEE Sensors Journal, vol. 18, no. 11, pp. 4378-4383, 2018.

[39] https://phytonet-doo.business.site/

[40] https://www.sigmaaldrich.com/labware/syringe-filters.html

[41] https://www.sigmaaldrich.com/technicaldocuments/articles/biology/sterile-millex-filters-for-cell-culture.html

[42] V. F. Lvovich, "Impedance Spectroscopy: Applications to Electrochemical and Dielectric Phenomena", John Wiley \& Sons, 2012, p. 50.

[43] N. O. Weiss, H. Zhou, L. Liao, Y. Liu, S. Jiang, Y. Huang and X. Duan, "Graphene: An Emerging Electronic Material", Advanced Materials, vol. 24, no. 43, pp. 5782-5825, 2012.
[44] C. Yim, N. McEvoy, H.Y. Kim, E. Rezvani, and G.S. Duesberg, "Investigation of the interfaces in schottky diodes using equivalent circuit models", ACS applied materials \& interfaces, vol. 5, no. 15, pp. 69516958. 2013, doi: 10.1021/am400963x

[45] M. Das, J. Datta, S. Sil, A. Dey, R. Jana, S. Halder, and P. P. Ray, "Equivalent circuit analysis of $\mathrm{Al} / \mathrm{rGO}-\mathrm{TiO} 2$ metal-semiconductor interface via impedance spectroscopy: Graphene induced improvement in carrier mobility and lifetime", Materials Science in Semiconductor Processing, vol. 82, pp.104-111, 2018, doi: 10.1016/j.mssp.2018.03.039

[46] H. Schiweck, A. Bär, R. Vogel, E. Schwarz, M. Kunz, B. Lüssem, M. Moser, and S. Peters, "Sugar alcohols", in Ullmann's encyclopedia of industrial chemistry, Wiley-VCH Verlag $\mathrm{GmbH}$ \& Co. KGaA, GermanyEditors: Wiley-VCH, 2000 doi: 10.1002/14356007.a25_413.pub2

[47] A. N. Soriano, K. G. R. Dollente, R. J. D. Tabaquero, and A. P. Adornado, "Estimation of diffusion coefficients of ascorbate-based ions at infinite dilution through electrolytic conductivity measurements", In IOP Conference Series: Earth and Environmental Science, vol. 191, no. 1, p. 012028. 2018, doi: 10.1088/1755-1315/191/1/012028

[48] A. C. R. Neto, R. F. Pires, R. A. Malagoni and M. R. Franco Jr, "Solubility of vitamin C in water, ethanol, propan-1-ol, water+ ethanol, and water+ propan-1-ol at (298.15 and 308.15) K", Journal of Chemical \& Engineering Data, vol. 55, no. 4, pp. 1718-1721, 2010 doi: $10.1021 / \mathrm{je} 900687 \mathrm{y}$

[49] National Center for Biotechnology Information. PubChem Compound Summary for CID 54670067, Ascorbic acid. https://pubchem.ncbi.nlm.nih.gov/compound/Ascorbic-acid

[50] T. Leveling, "The relationship between $\mathrm{pH}$ and conductivity in a Lithium Contaminated, De-ionized water system", Pbar Note 675, 2002.

[51] A. Kaplan, Z. Yuan, J. D. Benck, A. G. Rajan, X. S. Chu, Q. H. Wang and M. S. Strano, "Current and future directions in electron transfer chemistry of graphene", Chem. Soc. Rev., vol. 46, pp. 4530-4571, 2017, doi: $10.1039 / \mathrm{c} 7 \mathrm{cs} 00181 \mathrm{a}$ 